\title{
Treatment of Radon Rich Bottled Water by Granular Activated Carbon Adsorption Method
}

\author{
Saddig D. Jastaniah, Bassam Z. Shakhreet, Hanan Y. Abbas, Awad M. Elkhadir, Saeed M. Bafaraj \\ Department of Diagnostic Radiology, Faculty of Applied Medical Sciences, King Abdulaziz University, Jeddah, KSA \\ Email: sjastaniah@kau.edu.sa
}

Received November 13, 2013; revised December 9, 2013; accepted December 16, 2013

Copyright (C) 2014 Saddig D. Jastaniah et al. This is an open access article distributed under the Creative Commons Attribution License, which permits unrestricted use, distribution, and reproduction in any medium, provided the original work is properly cited. In accordance of the Creative Commons Attribution License all Copyrights (C) 2014 are reserved for SCIRP and the owner of the intellectual property Saddig D. Jastaniah et al. All Copyright (C) 2014 are guarded by law and by SCIRP as a guardian.

\section{ABSTRACT}

We explore the use of Granular Activated Carbon (GAC) as a mitigation technique for the treatment of drinking water that was artificially enriched with Rn-222 in laboratory by placing a radium rich granite stone (pitchblende) in a closed container filled with tap water for several days in order to allow Rn-222 concentration to approach its highest possible level. Granular Activated Carbon (GAC) was immersed into the water container in order to adsorb Rn-222 in radon-rich water. The optimum activation procedure for GAC preparation was determined. Suitable contact time and optimum quantities of GAC needed to reduce Rn-222 concentration in water were experimentally determined. There is a much greater increase in $\mathbf{R n}-222$ removal from water in the ranges from 1 part: 1000 parts to 4 parts: 1000 parts GAC/water ratios. Further increase in GAC/water ratio slowly affects the $\mathrm{Rn}-222$ removal.

\section{KEYWORDS}

Radon; Mitigation; Drinking Water; GAC

\section{Introduction}

The treatment of Rn-222 rich water by suitable mitigating techniques is necessary for cases of well water containing elevated level of Rn-222, since there is an increasing evidence correlating ingestion of $\mathrm{Rn}-222$ rich water and the potential for induction of internal cancers $[1,2]$.

The Rn-222 removal from water by different treatment processes, such as Granular Activated Carbon (GAC) adsorption, and various forms of aeration was described [3].

Dixon and Lee (1989) [4] reported that GAC is a very effective mitigating technique. It depends, however, on the contact time with the carbon bed. In spite of its effectiveness, the method is not applied to many systems because of operational and economical considerations. In addition to these considerations, the formation of lowlevel radioactive waste (Spent GAC) requires waste disposal processes. Aeration as a mitigating technique was also tested and found to be very effective and economical.
GAC removal method was discussed by Lowry (1983) [5]. From the operational standpoint, the GAC is used for the removal of $\mathrm{Rn}-222$ from three small water supply systems in order to determine its efficiency (Dixon and Lee 1988) [6].

On the other hand, Clifford et al. (1988) [7] found that GAC adsorbing efficiency for removing Rn-222 from drinking water decreased with extensive use. They reported that GAC columns were added to a water treatment system in order to remove the Rn-222. The initial removal was $64 \%$, and it decreased to $20 \%$ after one month of continuous operation.

A comparative study of different types of granular activated carbon in removing medium level radon from water was investigated by Abdulrahman I. Alabdula'aly and Hamed B. Maghrawy (2011) [8]. This study is useful for designing a GAC adsorption system for the removal of medium level radon concentration from water supplies.

The disposal of spent granular activated charcoal (GAC) filters used in the application of residential radon reduc- 
tion from well water may one day become a concern [9]. Residual radioactivity collected on the filter media, such as natural uranium, radium, and lead needs to be accounted for in order to minimize problems at the disposal site. Well water is known to contain small quantities of uranium, radium, and radon, and the GAC filter has varying collection efficiencies for each. Lead, a decay product of radon will also build up on the filter. Various disposal concerns need to be considered such as US DOT transportation issues, US NRC regulations, and state TENORM guidance. This paper addresses these issues, in particular as they relate to Pennsylvania.

During the past few years, the use of granular activated carbon (GAC) filters for removing radon from water has rapidly increased in Finland [10]. However, in many cases the risks related to external gamma radiation originating from the filter during its use are not appropriately considered. In some cases, the filter has caused significant dose rates in the living areas of a home. It was decided to prepare technical guidance on installing a GAC filter in a safe manner. Radiological criteria for gamma dose were established and used for deriving design parameters regarding appropriate shielding and safety distances. Because of the related risks, however, today it is recommended that GAC filters should not be installed in homes. The only exception is that installations do not require any specific considerations concerning external gamma radiation, for example, cases where the filter is placed underground into a well.

It can be seen that GAC treatment is an effective and economical method if it is used for small water supply systems, where the required quantity of GAC is small.

\section{Treatment of Rn-222 Rich Water by Granular Activated Carbon (GAC) Adsorption Method}

GAC has been used as a means of water purification to remove taste and odor from public water supplies since early this century without harmful effects. Recently it became widely used for household needs. The interesting part is that GAC also has the ability to adsorb Rn-222 and other isotopes such that it could lower radon content in radon rich water to very low concentrations, as reported by Lowry 1983 [5].

In order to evaluate the GAC method under controlled laboratory conditions, several experiments were conducted on GAC radon adsorption under specified conditions. But before outlining the details of these experiments, the parameters affecting GAC activation have to be investigated in order to adopt the optimum conditions for activation.

\subsection{Materials and Equipment}

The materials needed to perform GAC adsorption expe- riments include: Rn-222 rich water, high efficiency mineral oil scintillator, borosilicate glass vials of $20 \mathrm{ml}$ capacity with foil lined caps, glass cylinders, beakers, plastic bottles of $1000 \mathrm{ml}$ capacity, and carbon. The equipment used are liquid scintillation counter (type LSC2), connected to a scalar rate meter (type SR8).

\subsection{Procedures and Methods}

\subsubsection{The Optimum Activation Procedures for GAC}

The activation procedures that will be adopted in this work are determined by finding out the suitable activation temperature and time for GAC which will be used in the $\mathrm{Rn}-222$ rich water treatment experiments. This was achieved by performing several activation experiments.

1) Optimizing activation temperature

In order to determine the optimum activation temperature, the variation in adsorption capacity of GAC activated at different temperatures was tested. Five grams of GAC were subjected to activation in an oven for 2 hours, at $110^{\circ} \mathrm{C}$. A similar quantity was also activated but this time at $150^{\circ} \mathrm{C}$. Both activated GAC samples were allowed to cool over night in the oven (dry atmosphere). This was followed by mixing each of the carbon samples with 500 $\mathrm{ml}$ of $\mathrm{Rn}-222$ rich water of known concentration for a contract time of 1 hour.

2) Optimizing activation time

After optimizing the activation temperature at $150^{\circ} \mathrm{C}$, which will be used in the GAC adsorption experiments, the optimum activation time was also determined by studying variation in the adsorption capacity of GAC with variation in the duration of the heat treatment at $150^{\circ} \mathrm{C}$. Five grams of GAC were subjected to activation in an oven for 1 hour, at $150^{\circ} \mathrm{C}$. Similar quantities were also activated for $1.5,2,3$ and 5 hours. They were allowed to cool in the oven over night. Each of the carbon samples was then added to $500 \mathrm{ml}$ of $\mathrm{Rn}-222$ rich water of known concentration for a contract time of 1 hour.

\subsubsection{Use of GAC in Radon Removal}

Study of Rn-222 removal from water by adsorption of $\mathrm{Rn}-222$ atoms on the surface of the GAC is the purpose of the following experiments. In order to achieve this purpose, the factors affecting Rn-222 removal from water using GAC must be experimentally studied. These factors include: quantities of GAC used, contact time between water and carbon, and volume of the treated water.

\subsubsection{Factors Affecting Rn-222 Removal from Water Using GAC Method \\ 1) Quantities of GAC}

The quantity of GAC is a very important factor affecting Rn-222 removal from water. Several masses of GAC were used (1 g, $2 \mathrm{~g}$, and $5 \mathrm{~g}$ ) for treatment of each of the tested water volumes $(250 \mathrm{ml}, 500 \mathrm{ml}$, and $1000 \mathrm{ml})$. 
Three plastic bottles were filled with equal volumes of Rn-222 enriched water, and GAC was added. A plastic bottle was also filled with the same volume of water, but without adding any GAC so as to act as a control. Samples were then taken from all the bottles at the end of selected contact times $(0.5,1,2,6,12,24 \mathrm{hrs})$. The samples taken from the control bottle were used to correct for the decay calculations of Rn-222 during each testing contact time. It should be noted that the bottles were capped with an air tight cap during the duration of the experiments.

\section{2) Contact time}

The contact time or so called bed time is the time duration of the contact between GAC and water sample to certain volume. The selected contact times were $(0.5,1,2$, 6, 12, $24 \mathrm{hrs}$ ).

A $250 \mathrm{ml}$ of Rn-222 rich water of known concentration was mixed with 5 grams of GAC for a contact time of $1 \mathrm{hr}$. Samples were then taken to determine the fraction of Rn-222 remaining. The carbon was reactivated and reused again. This was repeated eight times under identical conditions.

3) Determination of R-222 half life from used GAC

In order to confirm the adsorption of radon by GAC, a growth curve for Rn-222 daughters and a half life measurement for radon were done.

The experiments were done by taking $50 \mathrm{ml}$ of Rn-222 rich water and mixing it with 5 grams of GAC and placing the mixture in an air tight capped bottle. After one hour of contact time the GAC was removed and immediately placed in another air tight capped bottle was shaken for 5 minutes. Samples were then taken in a scintillation vial at 1, 2, 3, 5, 10, 12 and 24 hrs. The measured count rates were used to plot a growth curve for Rn-222 daughters as shown in Figure 1. The maximum count rate reached was $1530 \mathrm{cpm}$. The half life measurement for Rn-222 in the scintillation liquid was done on the liquid scintillation sample taken at $24 \mathrm{hr}$. post mixing with the used GAC. The activity in the liquid scintillator was followed for several days (about 4 half lives) and a decay curve was plotted as shown in Figure 2. The Rn222 half life was calculated from the equation of the decay curve to be equal to 4.15 days. This value had an error of $8 \%$ from the true value (3.825 days).

\section{Results and Discussion}

\subsection{Optimum Activation Procedure for GAC}

The optimum activation temperature for GAC is $150^{\circ} \mathrm{C}$ and the optimum activation time is 2 hours as shown in Figures 3 and 4, respectively. GAC was allowed to cool in a dry environment, and then it was used shortly after it had reached room temperature.

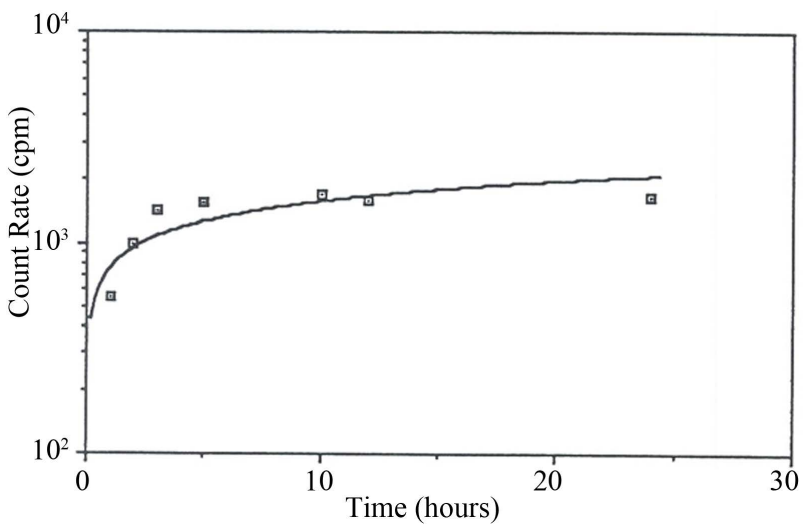

Figure 1. The growth curve of Rn-222 daughters in liquid scintillator oil.

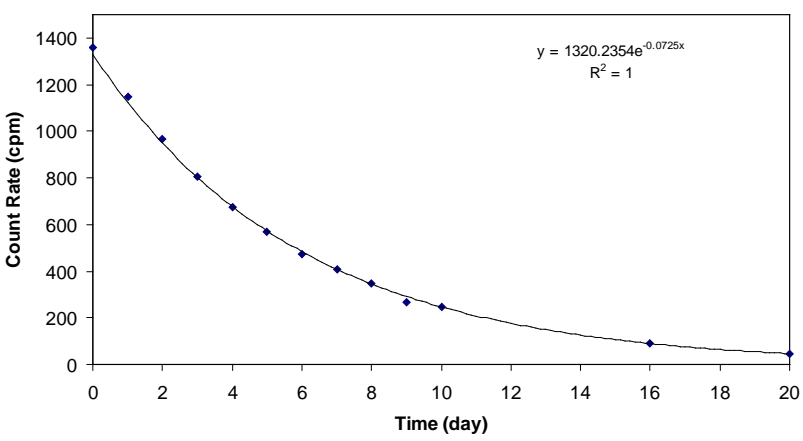

Figure 2. The decay curve of $\mathbf{R n}-222$ present in scintillator oil.

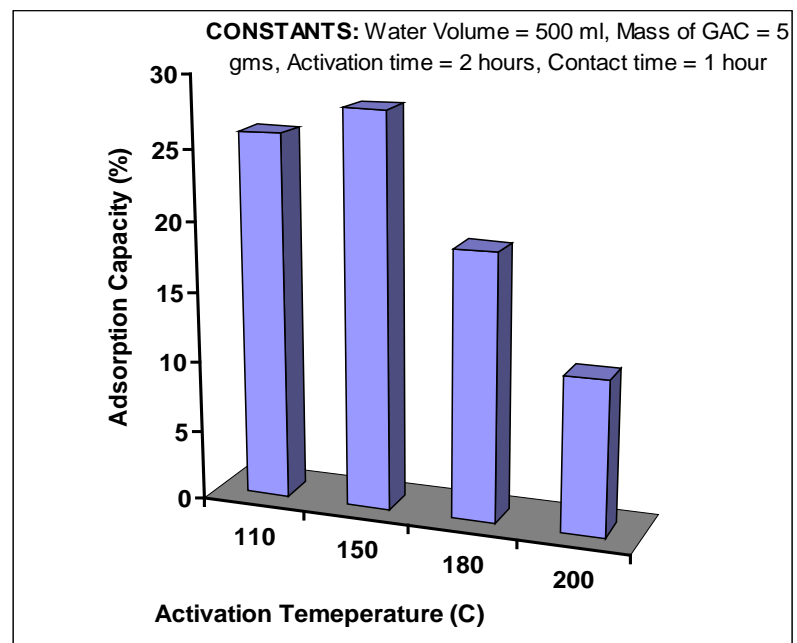

Figure 3. The variation in adsorption capacity of GAC activated at different temperatures.

\subsection{Efficiency of Rn-222 Removal at Different GAC/Water Ratios}

Figure 5 presents the results of radon removal at different GAC/water ratios determined from 3 repeated experiments. The Figure shows that there is a much greater increase in Rn-222 removal from water in the ranges 
from 1 part: 1000 parts to 4 parts: 1000 parts GAC/water ratios. Further increase in GAC/water ratio slowly affects the $\mathrm{Rn}-222$ removal. The values of the relative $\mathrm{Rn}-222$

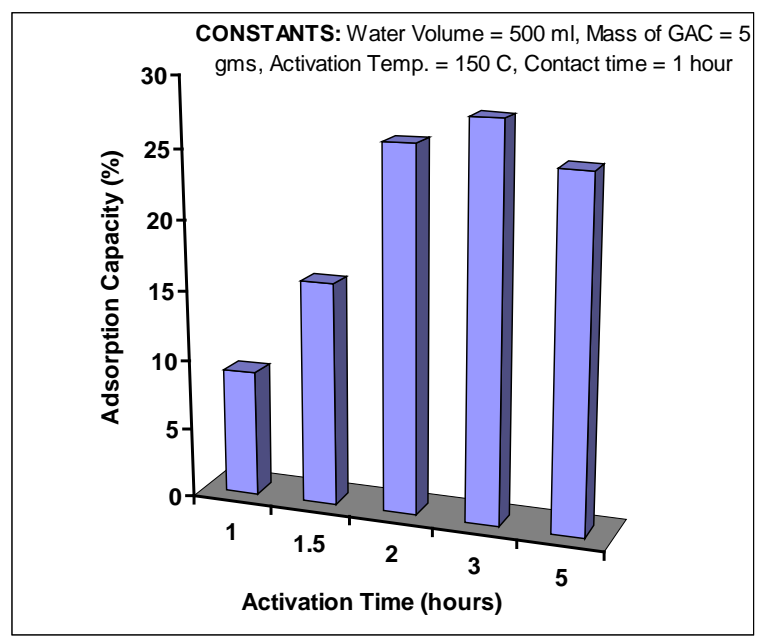

Figure 4. The variation in adsorption capacity of GAC activated at different times. removal are corrected for the physical decay of Rn-222. The achieved results are compatible with results of similar studies by Kinner et al. (1990) [3] and Dixon and Lee (1989) [4]. Those studies recommended the use of GAC whenever the treated water volume is small, so that least amounts of GAC will be required for Rn-222 removal. This is very important in order to keep the running cost of the treatment facility as low as possible. Furthermore the use of GAC system causes the carbon filters to become contaminated with pb-210 (a long living daughter of Rn-222). Hence, the GAC will eventually become radioactive waste that needs to be managed properly.

\subsection{Factors Affecting the Efficiency of GAC Method}

Results of samples that were taken from the treated water selected contact times (0.5, 1, 2, 6, 12 and 24 hours) are tabulated in Table 1 . The relative amounts of Rn-222 present in water decreases with increase in contact time. This was further represented by the curves shown in Figure 6.

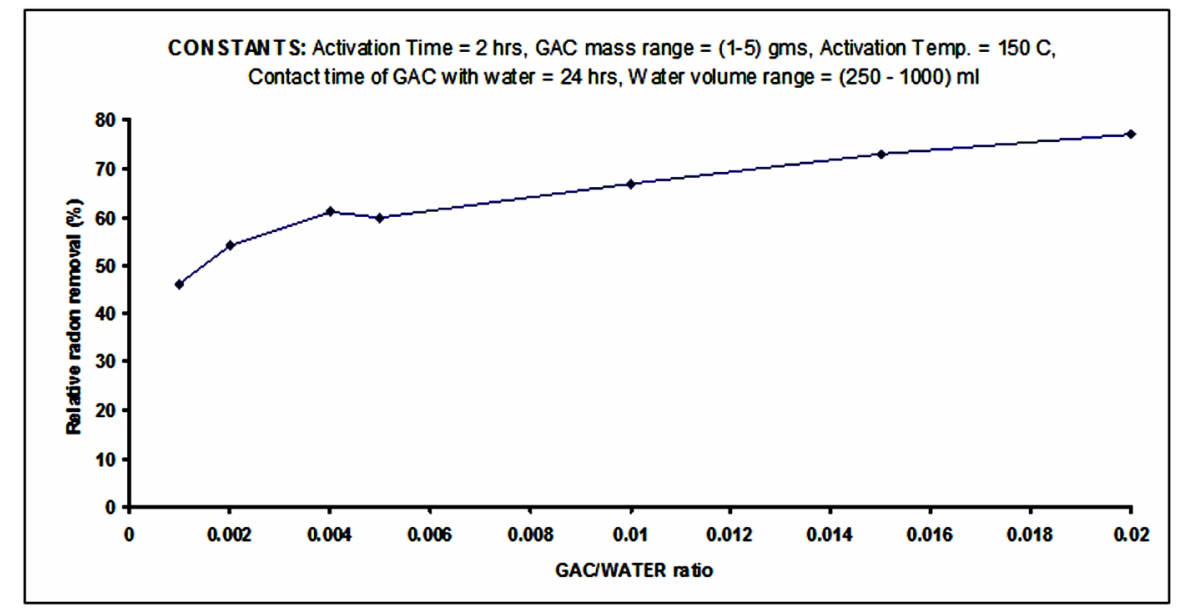

Figure 5. Rn-222 removals at different GAC/WATER ratios.

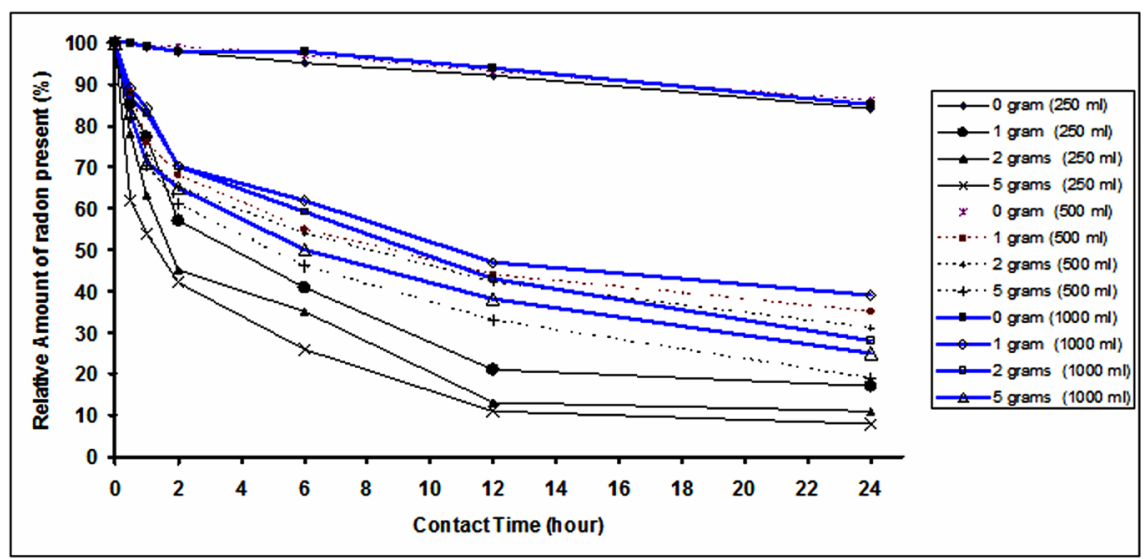

Figure 6. Removal of radon by different quantities of GAC from different volumes of radon rich water. 
Table 1. Summery of results of $\mathbf{R n}-222$ removal from water using GAC method.

\begin{tabular}{|c|c|c|c|c|c|c|c|c|}
\hline \multirow{2}{*}{$\begin{array}{c}\text { GAC Mass } \\
\text { (g) }\end{array}$} & \multirow{2}{*}{ Contact Time (hr) } & 0 & 0.50 & 1 & 2 & 6 & 12 & 24 \\
\hline & & \multicolumn{7}{|c|}{ Relative Radon Remainder (\%) } \\
\hline 0 & \multirow{4}{*}{$\begin{array}{l}\text { Water Volume } \\
250 \mathrm{ml}\end{array}$} & 100 & 100 & 99 & 98 & 95 & 92 & 84 \\
\hline 1 & & 100 & 85 & 77 & 57 & 41 & 21 & 17 \\
\hline 2 & & 100 & 78 & 63 & 45 & 35 & 13 & 11 \\
\hline 5 & & 100 & 62 & 54 & 42 & 26 & 11 & 8 \\
\hline 0 & \multirow{4}{*}{$\begin{array}{l}\text { Water Volume } \\
500 \mathrm{ml}\end{array}$} & 100 & 100 & 99 & 99 & 97 & 93 & 86 \\
\hline 1 & & 100 & 88 & 76 & 68 & 55 & 44 & 35 \\
\hline 2 & & 100 & 85 & 73 & 65 & 54 & 42 & 31 \\
\hline 5 & & 100 & 52 & 70 & 61 & 46 & 33 & 19 \\
\hline 0 & \multirow{4}{*}{$\begin{array}{l}\text { Water Volume } \\
1000 \mathrm{ml}\end{array}$} & 100 & 100 & 99 & 98 & 98 & 94 & 85 \\
\hline 1 & & 100 & 89 & 84 & 70 & 62 & 47 & 39 \\
\hline 2 & & 100 & 87 & 83 & 70 & 59 & 43 & 28 \\
\hline 5 & & 100 & 83 & 71 & 65 & 50 & 38 & 25 \\
\hline
\end{tabular}

The upper curves in Figure represent the results of natural decay of Rn-222 in the bottles not containing any GAC. This is used to correct for the reduction in Rn-222 concentration not due to addition of any GAC. By comparing these curves, it is clear that while enlarging the volume of water the GAC quantity becomes less effective in adsorbing the Rn-222, which is similar to the result reported by Lowry (1987) [11]. After 24 hours of contact time, the relative amounts of Rn-222 present in each of treated volumes (250, 500 and $1000 \mathrm{ml})$ in contact with 5 grams of GAC are $8 \%, 19 \%$ and $25 \%$ respectively. These values were upgraded to $23 \%$, 33\% and $40 \%$ after correcting for $\mathrm{Rn}-222$ reduction due to physical decay.

\subsection{Efficiency of Regenerated GAC}

The re-activation of carbon at $150^{\circ} \mathrm{C}$ for 24 hours and reuse did not affect its capacity for adsorbing Rn-222 very much. Eight reactivations or so called regenerations of used GAC were done and its capacity for radon adsorption was tested in the usual manner as shown in Figure 7. The GAC adsorption capacity was slightly varied for every regeneration, but GAC was still effective in $\mathrm{Rn}-222$ removal from water when reused several times. In a similar investigation by (Clifford et al. 1988) [7], GAC adsorption capacity was depleted with extensive use. It should be noted however that GAC accumulates long living radioactivity as reported by Dixon and Lee (1989) [4], thus it should be replaced after repeated use, since it can become a hazardous source of gamma radiation.

\section{Summary and Conclusions}

The granular activation carbon (GAC) filtration method was tested. The removal of Rn-222 from artificially

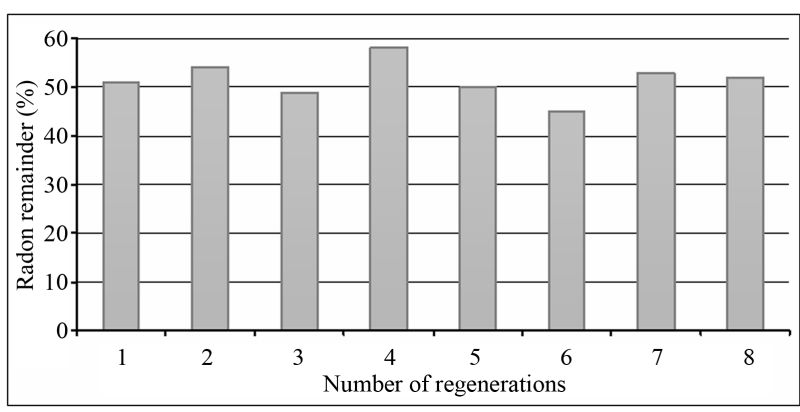

Figure 7. Regeneration of GAC. Constants: (contact time = $1 \mathrm{hr}$, water volume $=250 \mathrm{ml}$, mass of GAC $=5$ grams, activation Temp. $=150^{\circ} \mathrm{C}$, activation time $=2 \mathrm{hrs}$ ).

enriched water using this method reached about $78 \%$. Heat was found to have an effective ability for removing $\mathrm{Rn}-222$ from water. Measured removal of $\mathrm{Rn}-222$ from water that was kept at a maintained temperature of $39^{\circ} \mathrm{C}$ for 5 hours reached about $76 \%$.

Removal of Rn-222 from water by GAC will be enhanced by following the optimum activation process. The optimum activation temperature was found to be $150^{\circ} \mathrm{C}$, and the optimum activation time was found to be 2 hours. The optimum amount of GAC needed for removal of $\mathrm{Rn}-222$ from water is a function of treated water volume and contact time between water and GAC.

Finally, regeneration of GAC enables its repeated use. The radioactivity built up in GAC filters is the prime limit for the process of regeneration. Since GAC filters are expensive, it is recommended for applications requiring little amounts of GAC.

\section{REFERENCES}

[1] F. F. Cross, N. H. Harley and W. Holemann "Health Effects and Risk from Rn-222 in Drinking Water,” Health Physics, Vol. 48, No. 5, 1985, pp. 649-670. http://dx.doi.org/10.1097/00004032-198505000-00006

[2] D. J. Crawford-Brown, "Analysis of the Health Risk from Ingested Radon,” Lewis Publishers, Inc., Chelsea, 1990, pp. 17-25.

[3] N. E. Kinner, P. A. Quern, G. S. Schell, C. E. Lessard and J. A. Clement, "Treatment Technology for Removing Rn222 from Small Community Water Supplies,” Lewis Publishers, Inc., Chelsea, 1990, pp. 39-52.

[4] K. L. Dixon and R. G. Lee, "Radon in Groundwater Supplies,” Water Well Journal, Vol. 43, No. 6, 1989, p. 44.

[5] J. D. Lowry, "Removal of Rn-222 from Water Using Granular Activated Carbon Adsorption,” National Technical Information Service, Land and Water Resources Center Completion Representative, University of Maine, Orono., 1983, p. 1.

[6] K. L. Dixon and R. G. Lee, "Occurrence of Radon in Well Supply,” Journal-American Water Works Association, Vol. 80, No. 7, 1988, pp. 65-70.

[7] D. Clifford, W. Vijjeswarapu and S. Subramonian, "Eval- 
uating Various Absorbents and Membranes for Removing Radon from Ground Water," Journal-American Water Works Association, Vol. 80, No. 7, 1988, pp. 94-104.

[8] A. I. Alabdula'aly and H. B. Maghrawy, "Comparative Study of Different Types of Granular Activated Carbon in Removing Medium Level Radon from Water,” Journal of Radioanalytical and Nuclear Chemistry, Vol. 287, No. 1, 2011, pp. 77-85. http://dx.doi.org/10.1007/s10967-010-0804-1

[9] R. K. Lewis, "Disposal of Granular Activated Charcoal Used for the Treatment of Radon-222 in Well Water," Proceedings of the American Association of Radon Scien- tists and Technologists, International Symposium, Las Vegas, 2008.

[10] M. Markkanen, "Technical Guidance on Safe Installation of a GAC Filter Used for Removing Radon from Water," Journal of Radioactivity in the Environment, Vol. 7, 2005, pp. 665-669.

http://dx.doi.org/10.1016/S1569-4860(04)07081-0

[11] J. D. Lowry, W. F. Brutsaert, T. McEnerney and C. Molk, "Point-of-Entry Removal of Radon From Drinking Water," Journal-American Water Works Association, Vol. 79, No. 4, 1987, pp. 162-169. 